\title{
The load-balanced multi-dimensional bin-packing problem
}

\section{Trivella, Alessio; Pisinger, David}

\section{Published in:}

Computers and Operations Research

Link to article, DOI:

10.1016/j.cor.2016.04.020

Publication date:

2016

Document Version

Peer reviewed version

Link back to DTU Orbit

Citation (APA):

Trivella, A., \& Pisinger, D. (2016). The load-balanced multi-dimensional bin-packing problem. Computers and Operations Research, 74, 152-164. https://doi.org/10.1016/j.cor.2016.04.020

\section{General rights}

Copyright and moral rights for the publications made accessible in the public portal are retained by the authors and/or other copyright owners and it is a condition of accessing publications that users recognise and abide by the legal requirements associated with these rights.

- Users may download and print one copy of any publication from the public portal for the purpose of private study or research.

- You may not further distribute the material or use it for any profit-making activity or commercial gain

- You may freely distribute the URL identifying the publication in the public portal

If you believe that this document breaches copyright please contact us providing details, and we will remove access to the work immediately and investigate your claim 


\title{
The load-balanced multi-dimensional bin-packing problem
}

\author{
Alessio Trivella ${ }^{\mathrm{a}, *}$, David Pisinger ${ }^{\mathrm{a}}$ \\ ${ }^{a}$ Department of Management Engineering, Technical University of Denmark, \\ Produktionstorvet, Building 426, DK-2800 Kgs. Lyngby, Denmark
}

\begin{abstract}
The bin-packing problem is one of the most investigated and applicable combinatorial optimization problems. In this paper we consider its multi-dimensional version with the practical extension of load balancing, i.e. to find the packing requiring the minimum number of bins while ensuring that the average center of mass of the loaded bins falls as close as possible to an ideal point, for instance the center of the bin. We formally describe the problem using mixed-integer linear programming models, from the simple case where we want to optimally balance a set of items already assigned to a single bin, to the general balanced bin-packing problem. Given the difficulty for standard solvers to deal even with small size instances, a multi-level local search heuristic is presented. The algorithm takes advantage of the Fekete-Schepers representation of feasible packings in terms of particular classes of interval graphs, and iteratively improves the load balancing of a bin-packing solution using different search levels. The first level explores the space of transitive orientations of the complement graphs associated with the packing, the second modifies the structure itself of the interval graphs, the third exchanges items between bins repacking proper n-tuples of weakly balanced bins. Computational experiments show very promising results on a set of 3D bin-packing instances from the literature.
\end{abstract}

Keywords: multi-dimensional bin-packing, load balancing, MILP modeling,

\footnotetext{
* Corresponding author

Email addresses: atri@dtu.dk (Alessio Trivella), dapi@dtu.dk (David Pisinger)
} 
local search

\section{Introduction}

The bin-packing problem is one of the most investigated and applicable models in combinatorial optimization. The problem consists of packing objects of different sizes into a finite number of similar bins/containers, in a way that the

5 number of used bins is minimized. The bin-packing problem can be seen as a special case of the cutting stock problem, in which each item has also a demand, i.e. it must appear a specific number of times in the bins. There exist practical applications of the bin-packing problem in a broad range of disciplines, such as transportation and logistics, computer science, engineering, economics and manufacturing. For the variety and importance of its applications, the binpacking problem has been intensively studied and several interesting ideas for solving it have arisen during the years.

In some real-life situations, mostly related to the two- and three-dimensional cases, we are not only interested in determining a packing with fewest bins,

${ }_{15}$ but also obtaining well-balanced packings. Consider for example the problem of arranging items into an aircraft cargo area such that the center of mass, or barycenter, of the loaded plane falls as close as possible to an ideal point given by the aircraft's specifications. The position of the barycenter has an impact on the flight performance in terms of safety and efficiency, and even a minor displacement from the ideal barycenter can lead to a high increase of fuel consumption [1. Similar considerations apply in the loading of trucks and container ships.

Packing and balancing a set of items represent two conflicting objectives and in this paper we will investigate how to integrate them into a single problem. The goal is to arrange the items in the smallest number of bins, while ensuring the best overall load balancing of the used bins. 


\subsection{Related Literature}

In the following we present the essential literature along two main lines: the classical multi-dimensional bin-packing problem and variants based on load balancing.

The literature about multi-dimensional bin-packing is vast and a huge number of heuristic algorithms and dedicated exact procedures have been developed. Martello and Vigo 2] proposed the first exact method for the 2D bin-packing problem using a two-level Branch-and-Bound method. Using a similar procedure, Martello et al. [3] described an exact algorithm for the 3D case and introduced the discrete set of Corner Points to reduce the search space.

Fekete and Schepers [4, 5] defined an implicit representation of multi-dimensional packings by means of interval graphs, built by checking whether the projections of items on the coordinate axes are pairwise overlapping. With such character-

40 ization the authors successfully developed a two-level search tree.

Several promising heuristics are based on a local search framework. Lodi et al. [6] proposed a unified tabu search, called TSpack, which can be used for solving many variants of any multi-dimensional bin-packing problem. Faroe et al. [7] presented the Guided Local Search method where memory guides the search to

45 promising regions of the solution space.

Crainic et al. 8] developed constructive heuristics based on an efficient way to place items into a bin, exploiting the free volume better than previous methods. This is done introducing the Extreme Points, which extend the concept of Corner Points. The same authors presented the $T S^{2}$ pack [9], a two-level tabu search algorithm which, among others, makes use of the graph representation of Fekete and Schepers to verify the feasibility of a packing and developed GASP [10], a meta-heuristic framework for solving the multi-dimensional bin-packing which combines the simplicity of greedy algorithms with learning mechanisms. Other heuristic approaches for multi-dimensional bin-packing problems can be 55 found in Egeblad and Pisinger [11, Lodi et al. [12], Pisinger and Sigurd [13]. In recent years some authors started to examine different packing-related problems involving the load balancing of items. The literature is however still quite 
scarce, perhaps due to the fact that considering the load balancing adds further complexity to the already $\mathcal{N} \mathcal{P}$-hard packing problems. Davies and Bischoff [14] and Junqueira et al. [15] considered load balancing in the $3 \mathrm{D}$ container loading problem. Paquay et al. [16] provided a formulation of 3D bin-packing deriving from an air cargo application which takes into account weight distribution constraints. Mongeau, Bes [1] and Kaluzny and Shaw [17] also focused on the optimal aircraft load balancing. In papers [1, 14, 15, 16, 17. the target is balancing a single bin and specific physical constraints related to for example aircraft's specifications are included in the models.

In some papers the load balancing is handled by imposing the center of mass to lie inside "stable" region. Baldi et al. [18, analyze a 3D knapsack problem with these kinds of balancing constraints, whereas de Queiroz and Miyazawa [19] consider the 2D strip packing.

Using the concept of a stable region for a bin-packing problem imposes some challenges. It may happen that no bin-packing solution using the smallest number of bins respects the balancing constraints, especially if the safe region is very restricted. On the other hand, large regions allow a feasible barycenter too far away from an ideal point, and in industrial situations it translates into an increase of costs or fuel consumption. Even though a stable region is relatively easy to model, we will also investigate other ways of modeling the balance constraints.

Some authors model the load balancing with a multi-objective formulation aimed to simultaneously minimize the number of used bins and the load imbalance of the bins, frequently solved using meta-heuristics. In particular, the 2D multi-objective bin-packing is solved by Fernández et al. 20. using a parallel memetic algorithm, and by Liu et al. 21] with evolutionary particle swarm optimization. Imai et al. 22 consider the load balancing and stability factors 85 for a shipping cargo with a particular cellular structure to place containers. 


\subsection{Recalling the bin-packing problem}

The multi-dimensional bin-packing problem (MBP) can be formulated using a mixed-integer linear programming (MILP) model. Assume a set of $n$ rectangularshaped boxes indexed over $V=\{1, \ldots, n\}$ is given in a $D$-dimensional space, $D \in \mathbb{N}$, and box $i$ has sizes $w_{i d}$ for $d \in D$. Infinitely many bins are available, having identical sizes $W_{d}$. The goal is to orthogonally pack all boxes into the bins avoiding overlap and using as few bins as possible. Rotation of items is not allowed.

For each $i \in V, d \in D$, the variable $x_{i d} \in \mathbb{R}$ represents the coordinate of the lower face of item $i$ in direction $d$, while the lower face of the bins is assumed to have coordinate 0 in every direction. The integer variable $a_{i}$ denotes the number of the bin containing item $i$. Then, for each couple of items $i, j \in V$ and $d \in D$, we introduce the binary variable $l_{i j d}=1$ if and only if box $i$ is located entirely lower than $j$ in direction $d$. Let $N$ denote the number of used bins, and lastly consider the binary variables $p_{i j}=1$ if and only if $a_{i}<a_{j}$. Thus, we can write the following model:

$\min N$

$$
\begin{aligned}
& \text { s.t. : } \sum_{d \in D}\left(l_{i j d}+l_{j i d}\right)+p_{i j}+p_{j i} \geq 1 \\
& \forall i<j \in V \\
& x_{i d}-x_{j d}+W_{d} l_{i j d} \leq W_{d}-w_{i d} \\
& \forall i \neq j \in V, d \in D \\
& x_{i d} \leq W_{d}-w_{i d} \\
& \forall i \in V, d \in D \\
& a_{i}-a_{j}+n p_{i j} \leq n-1 \\
& \forall i \neq j \in V \\
& 1 \leq a_{i} \leq N \\
& \forall i \in V
\end{aligned}
$$

Constraints (2) ensure that a pair of items $i, j$ in the same bin do not overlap, (3) translates the definition of $l_{i j d}$ in a mathematical form, (4) ensures that the bin's boundaries are not exceeded and (5) forces two overlapping items to lie in different bins.

The model would remain correct with $D=1$, but much simpler formulations 
exist in such case and we will limit our discussion to $D \geq 2$. Having a $D$ dimensional formulation of the problem makes it easy for us to treat the twoand three-dimensional cases simultaneously. Practical applications of the probshowed that many scheduling problems can be formulated as multi-dimensional bin packing problems.

Notice that the model makes use of a large number of big-M constraints which will loose their effect when solving the LP-relaxation, and thus bounds from the

\section{Modeling the load-balanced multi-dimensional bin-packing prob- lem}

The load-balanced multi-dimensional bin-packing problem consists in packing parallelepiped objects into the smallest number of bins, in such a way that the LP-relaxation are generally far from the integer optimal solution value. Moreover, the model contains many symmetric solutions, for example taking any permutation of the bins $a_{i}$. Constraints (5) have similarities with the MillerTucker-Zemlin constraints for the Traveling Salesman Problem [24] and the $a_{i}$ could be declared as real variables instead of integer but, because of the symmetry, without improving performance in a major way. For these reasons, even if we break some symmetries adding constraints as $a_{i} \leq i$, the model remains extremely difficult to solve in practice for standard MILP solvers, frequently failing even when dealing with instances constituting 30-35 items.

We have now all the elements to introduce the additional balancing requirement and describe the load-balanced multi-dimensional bin-packing problem. The remainder of the paper is structured as follows. In Section 2 we formulate a MILP model for the load-balanced multi-dimensional bin-packing problem. In Section 3 the exact theory is supported with the definition of a multi-level local search heuristic algorithm able to handle also large instances. Section 4 is dedicated to computational results and in Section 5 some conclusions are drawn.

center of mass of the loaded bins fall as close as possible to a desired location. 
In other words, the sum of displacements from the desired barycenter location over the (smallest number of) used bins is minimized.

To the best of our knowledge, no MILP model for such problem has been defined in the literature, thus, we will treat it in details. The explanation simplifies if we first consider the restricted problem where a single bin has to be balanced, and then extend to the general case.

\subsection{Balancing a single bin}

Suppose you have already obtained a feasible solution of the MBP using an exact or heuristic method, and consider a single bin. We want to arrange a set of $m$ given boxes $U=\{1,2, \ldots, m\}$ into the $D$-dimensional bin, so that the distance between the barycenter of the loaded bin and our ideal point inside the bin, for instance its geometrical center, is minimized. By assumption, the bin can accommodate all the items.

Assume that boxes are homogeneous and have densities $\rho_{i}, i \in U$. With $\rho_{i}$ we can determine the mass $m_{i}$ of box $i$ by $m_{i}=\rho_{i} \cdot v l_{i}$, where $v l_{i}=\prod_{d \in D} w_{i d}$ is the volume of box $i$. Equivalently, we could be given directly the masses $m_{i}$ instead of densities.

Concepts from physics, like density, mass and volume, normally refer to threedimensional objects, but will be abstractly used also in spaces of higher dimension.

Since box $i$ is homogeneous and rectangular-shaped, its barycenter coincides with its geometric center that corresponds to $C_{i}=\left(x_{i d}+w_{i d} / 2\right)_{d \in D}$, and the barycenter $B$ of the whole loaded bin can be computed using the expression:

$$
B=\left(B_{d}\right)_{d \in D}=\frac{\sum_{i \in U} m_{i} C_{i}}{\sum_{i \in U} m_{i}}
$$

Then, the objective function $f(x)=f\left(\left(x_{i d}\right)_{i, d}\right)$ represents the distance of the barycenter $B$ from its ideal location, denoted as $B^{o p t}=\left(B_{d}^{o p t}\right)_{d \in D}$. The $\mathscr{L}_{1^{-}}$ norm, or rectangular norm, i.e. the sum of the absolute values of the differences in the $D$ components, is used to compute the distance:

$$
f(x)=\left\|B^{o p t}-B\right\|_{1}=\sum_{d \in D}\left|B_{d}^{o p t}-B_{d}\right|
$$


The $\mathscr{L}_{1}$-norm is chosen since it better allows to separately manage the displacements in each direction and because it can be easily linearized.

Sometimes it can be more important to balance in one coordinate than the others. For example, someone might consider more relevant to obtain a perfect balancing on the $x$ and $y$ axes of a 3D container without caring too much about the height of the barycenter, or vice versa. As a consequence, some weights $k_{d} \geq 0$ are incorporated into the definition of $f$ in order to suit the individual needs to tune the balancing criteria, and a more general objective function looks like:

$$
f(x)=\sum_{d \in D} k_{d}\left|B_{d}^{o p t}-B_{d}\right|
$$

As an example, when loading a truck, it could be reasonable to choose the center of the base of the container as the ideal barycenter, i.e. the point with coordinates $\left(\frac{1}{2} W_{x}, \frac{1}{2} W_{y}, 0\right)$. If we assume that a low barycenter is twice as important as balancing in $x$ and $y$ directions, then the objective function will look like $\left|\frac{1}{2} W_{x}-B_{x}\right|+\left|\frac{1}{2} W_{y}-B_{y}\right|+2\left|B_{z}\right|$.

Coming back to the general case and substituting the components of $B$ with their full expressions, we obtain:

$$
f(x)=\sum_{d \in D} k_{d}\left|B_{d}^{o p t}-\frac{1}{M_{U}}\left(\sum_{i \in U} m_{i}\left(x_{i d}+\frac{w_{i d}}{2}\right)\right)\right|
$$

where $M_{U}=\sum_{i \in U} m_{i}$ is the total mass of boxes belonging to the set $U$.

The function $f$ is not linear since it contains absolute values. A well-known trick to handle an absolute value in the objective function is to introduce two additional positive support variables indicating the positive and negative parts of the quantity inside the absolute value. In our case we define $r_{d}, s_{d} \geq 0$ for $d \in D$ satisfying:

$$
r_{d}-s_{d}=B_{d}^{o p t}-\frac{1}{M_{U}}\left(\sum_{i \in U} m_{i}\left(x_{i d}+\frac{w_{i d}}{2}\right)\right)
$$

making it possible to rewrite the objective to:

$$
f(x)=\sum_{d \in D} k_{d}\left(r_{d}+s_{d}\right)
$$


Finally, reusing the set of variables $l_{i j d}$ already introduced for the MBP and the blocks of constraints ensuring no overlap and no violation of the boundaries, the problem is formulated using the following linear model:

$$
\begin{array}{lr}
\min \sum_{d \in D} k_{d}\left(r_{d}+s_{d}\right) \\
\text { s.t. }: r_{d}-s_{d}=B_{d}^{o p t}-\frac{1}{M_{U}}\left(\sum_{i \in U} m_{i}\left(x_{i d}+\frac{w_{i d}}{2}\right)\right) & \forall d \in D \\
\sum_{d \in D}\left(l_{i j d}+l_{j i d}\right) \geq 1 & \forall i<j \in U \text { (10) } \\
x_{i d}-x_{j d}+W_{d} l_{i j d} \leq W_{d}-w_{i d} & \forall i \neq j \in U, d \in D(11) \\
& x_{i d} \leq W_{d}-w_{i d} \\
\operatorname{var}: x_{i d}, r_{d}, s_{d} \in \mathbb{R}^{+} \quad l_{i j d} \in\{0,1\} & \forall i \in U, d \in D(12)
\end{array}
$$

We will refer to 813 as the multi-dimensional single load-balancing problem (SLB).

The structure is similar to the general MBP and most of the constraints are essentially the same. Hence, again, the large use of conditional constraints and big-M coefficients makes it difficult to solve the model in practice using standard solvers.

Notice that, by assumption, we start with a set of items which can be packed into one container, hence feasible solutions do exist. However, this information is not present a priori in the model, and if the number of boxes is large (more than 25-30) with a high filling of the bin's volume, then it can be hard for the solver even to find a feasible solution. Moreover, even when a feasible solution is found quickly, the gap from the best lower bound can take a huge amount of time to be closed.

\subsection{Packing and balancing}

Moving forward to the general problem, we can first assign items to the minimum number of bins by solving the MBP model, and next apply the SLB model to 
rearrange items inside each used bin in order to optimally balance their load. Unfortunately, this does not completely fulfill our task since the two models are not linked together and the packing and balancing phases are performed in an asynchronous manner. Indeed, the MBP has generally many different solutions and there may exist other assignments of items to the smallest number of bins which yield to an overall better balancing. In other words, the output of the packing phase can be a bad input for the balancing phase, and the solution we get after applying the two sequential phases is not guaranteed to be the optimal for our overall problem.

The task now is to combine the packing and balancing phases together and formulate an integrated MILP model.

\subsubsection{The objective function}

In the general case more bins are involved and the objective function will contain the sum of the displacements from the desired barycenter over all used bins. Let us denote by $U_{j}$ the set of boxes inside bin $j$. Remember that for a single bin $j$ the objective $f_{j}$ is modeled as:

$$
f_{j}(x)=\sum_{d \in D} k_{d}\left(r_{j d}+s_{j d}\right)
$$

where $r_{j d}+s_{j d}$ is the absolute value of the difference between the optimal location $B_{d}^{\text {opt }}$ and the $d^{\text {th }}$ coordinate $B_{j d}$ of the barycenter of bin $j$, i.e.:

$$
r_{j d}+s_{j d}= \begin{cases}\left|B_{d}^{o p t}-\frac{1}{M_{U_{j}}}\left(\sum_{i \in U_{j}} m_{i}\left(x_{i d}+\frac{w_{i d}}{2}\right)\right)\right| & \text { if bin } j \text { is used } \\ 0 & \text { otherwise }\end{cases}
$$

We will see later how to correctly handle such variables in a linear framework. The total displacement over all bins is then:

$$
f(x)=\sum_{j=1}^{n} f_{j}(x)=\sum_{j=1}^{n} \sum_{d \in D} k_{d}\left(r_{j d}+s_{j d}\right)=\sum_{d \in D} k_{d} \sum_{j=1}^{n}\left(r_{j d}+s_{j d}\right)
$$

where the sum is calculated over $j=1, \ldots, n$ since the number of items $n$ is an upper bound on the number of bins that will be used. 
By definition of the problem, the main goal is still to minimize the number of bins, and a solution that uses $N+1$ bins is considered worse than a solution that uses $N$, regardless of a better overall balancing of the first. Consequently, the objective function can be defined as:

$$
f(x)=C N+\sum_{d \in D} k_{d} \sum_{j=1}^{n}\left(r_{j d}+s_{j d}\right)
$$

where $C$ is chosen such that $C \geq \sum_{d \in D} k_{d} \sum_{j=1}^{n}\left(r_{j d}+s_{j d}\right)$, i.e. the component associated with the number of bins is larger than any possible value of the balancing part of the objective. In this way the balancing will not be in conflict with finding a solution using fewest bins. A possible value of $C$ is:

$$
C=\frac{1}{2} n \sum_{d \in D} k_{d} W_{d}
$$

This value can be refined in order to reduce the threshold value. For example, $n$ can be replaced with any upper bound on the smallest number of bins.

\subsubsection{New variables and constraints}

The variables used in the MBP model are unchanged. Furthermore, we introduce the binary variables:

$$
c_{i j}= \begin{cases}1 & \text { if } a_{i}=j, \text { i.e. item } i \text { is in bin } j \\ 0 & \text { otherwise }\end{cases}
$$

The implications $c_{i j}=1 \Longrightarrow a_{i}=j$ can be modeled with the following couples of constraints:

$$
\begin{array}{ll}
a_{i}-j \leq n\left(1-c_{i j}\right) & \forall i, j \in V \\
a_{i}-j \geq n\left(c_{i j}-1\right) & \forall i, j \in V
\end{array}
$$

To handle the reverse implication $a_{i}=j \Longrightarrow c_{i j}=1$, some additional support binary variables $\gamma_{i j}$ and $\delta_{i j}$ must be introduced together with the constraints:

$$
\begin{array}{ll}
a_{i}-j \leq-1+(n+1)\left(1-\gamma_{i j}\right) & \forall i, j \in V \\
a_{i}-j \geq 1-(n+1)\left(1-\delta_{i j}\right) & \forall i, j \in V \\
c_{i j}+\gamma_{i j}+\delta_{i j}=1 & \forall i, j \in V
\end{array}
$$


Now, the variables $c_{i j}$ are used to define the continuous variables $\alpha_{i j d}$ satisfying:

$$
\alpha_{i j d}= \begin{cases}m_{i}\left(x_{i d}+\frac{w_{i d}}{2}\right) & \text { if } c_{i j}=1 \\ 0 & \text { if } c_{i j}=0\end{cases}
$$

The implication $c_{i j}=1 \Longrightarrow \alpha_{i j d}=m_{i}\left(x_{i d}+\frac{w_{i d}}{2}\right)$ is ensured by imposing:

$$
\begin{array}{ll}
\alpha_{i j d}-m_{i}\left(x_{i d}+\frac{w_{i d}}{2}\right)+m_{i} W_{d} c_{i j} \leq m_{i} W_{d} & \forall i, j \in V, d \in D \\
m_{i}\left(x_{i d}+\frac{w_{i d}}{2}\right)-\alpha_{i j d}+m_{i} W_{d} c_{i j} \leq m_{i} W_{d} & \forall i, j \in V, d \in D
\end{array}
$$

and for $c_{i j}=0 \Longrightarrow \alpha_{i j d}=0$ we add the inequalities:

$$
0 \leq \alpha_{i j d} \leq c_{i j} W_{d} m_{i} \quad \forall i, j \in V, d \in D
$$

As we did for $\alpha_{i j d}$, we introduce the variables $\beta_{i j d}$ such that:

$$
\beta_{i j d}= \begin{cases}m_{i}\left(B_{d}^{o p t}-r_{j d}+s_{j d}\right) & \text { if } c_{i j}=1 \\ 0 & \text { if } c_{i j}=0\end{cases}
$$

This can be modeled through the families of constraints:

$$
\begin{array}{ll}
\beta_{i j d}-m_{i}\left(B_{d}^{o p t}-r_{j d}+s_{j d}\right)+m_{i} W_{d} c_{i j} \leq m_{i} W_{d} & \forall i, j \in V, d \in D \\
m_{i}\left(B_{d}^{o p t}-r_{j d}+s_{j d}\right)-\beta_{i j d}+m_{i} W_{d} c_{i j} \leq m_{i} W_{d} & \forall i, j \in V, d \in D \\
0 \leq \beta_{i j d} \leq c_{i j} W_{d} m_{i} & \forall i, j \in V, d \in D
\end{array}
$$

Finally, the following important equality

$$
\sum_{i \in V} \alpha_{i j d}=\sum_{i \in V} \beta_{i j d} \quad \forall j \in V, d \in D
$$

represents the load balancing on axis $d$ for bin $j$, stating that $r_{j d}+s_{j d}$ is the absolute value of the difference between $B_{d}^{o p t}$ and the $d^{t h}$ coordinate of the barycenter of bin $j$.

\subsubsection{The final model}

Summarizing the previous considerations, the resulting MILP model becomes 


$$
\begin{aligned}
& \min N C+\sum_{d \in D} k_{d} \sum_{j=1}^{n}\left(r_{j d}+s_{j d}\right) \\
& \text { s.t. : } \sum_{d \in D}\left(l_{i j d}+l_{j i d}\right)+p_{i j}+p_{j i} \geq 1 \\
& x_{i d}-x_{j d}+W_{d} l_{i j d} \leq W_{d}-w_{i d} \\
& x_{i d} \leq W_{d}-w_{i d} \\
& a_{i}-a_{j}+n p_{i j} \leq n-1 \\
& 1 \leq a_{i} \leq N \\
& n\left(c_{i j}-1\right) \leq a_{i}-j \leq n\left(1-c_{i j}\right) \\
& 1-(n+1)\left(1-\delta_{i j}\right) \leq a_{i}-j \leq-1+(n+1)\left(1-\gamma_{i j}\right) \\
& c_{i j}+\gamma_{i j}+\delta_{i j}=1 \\
& m_{i} W_{d}\left(c_{i j}-1\right) \leq \alpha_{i j d}-m_{i}\left(x_{i d}+w_{i d} / 2\right) \leq m_{i} W_{d}\left(1-c_{i j}\right) \\
& m_{i} W_{d}\left(c_{i j}-1\right) \leq \beta_{i j d}-m_{i}\left(B_{d}^{o p t}-r_{j d}+s_{j d}\right) \leq m_{i} W_{d}\left(1-c_{i j}\right) \\
& \alpha_{i j d}+\beta_{i j d} \leq 2 c_{i j} W_{d} m_{i} \\
& \sum_{i \in V} \alpha_{i j d}=\sum_{i \in V} \beta_{i j d}
\end{aligned}
$$$$
\forall i<j \in V
$$

$\forall i \neq j \in V, d \in D$

$\forall i \in V, d \in D$

$$
\forall i \neq j \in V
$$

$\forall i \in V$

$$
\forall i, j \in V
$$

$\forall i, j \in V$

$\forall i, j \in V$

$\forall i, j \in V, d \in D$

$\forall i, j \in V, d \in D$

$\forall i, j \in V, d \in D$

$\forall j \in V, d \in D$

$\forall i, j \in V, d \in D$ 
In the following we refer to (14 27) as LB-MBP.

The number of variables and constraints is greatly increased, although remaining $\mathcal{O}\left(n^{2}\right)$. In practice, this model is extremely difficult to solve even for small instances with 20-25 items.

\subsection{Comparison of the models. An example}

To clarify the previous concepts, consider the 3D instance with 16 items described in Table 1. The instance is generated from class 6 as defined in [3]. Bins are cubes of side 10 and the optimal barycenter location is $(5,5,0)$. We set the weights in the objective $k_{x}=k_{y}=k_{z}=1$ and densities $\rho_{i}=1$ for every $i \in V$.

\begin{tabular}{ccccccccccccccccc}
\hline item & 1 & 2 & 3 & 4 & 5 & 6 & 7 & 8 & 9 & 10 & 11 & 12 & 13 & 14 & 15 & 16 \\
\hline$w_{x}$ & 9 & 3 & 6 & 6 & 5 & 2 & 7 & 4 & 2 & 7 & 8 & 1 & 2 & 5 & 6 & 3 \\
$w_{y}$ & 7 & 5 & 6 & 1 & 3 & 3 & 3 & 8 & 7 & 10 & 6 & 2 & 4 & 4 & 9 & 7 \\
$w_{z}$ & 2 & 8 & 5 & 8 & 9 & 10 & 8 & 3 & 6 & 3 & 9 & 2 & 1 & 5 & 3 & 3 \\
\hline
\end{tabular}

Table 1: 3D instance with 16 items generated from class 6 (see [3]).

Let us compare the solutions obtained with CPLEX using the two following approaches:

- Sequential. Items are packed into the smallest number of bins using MBP and, afterwards, each bin is balanced to optimality using SLB.

- Integrated. The general model LB-MBP for packing and balancing is applied.

180

Using the sequential approach, the sixteen objects are optimally arranged into three bins as indicated in Table 2. In the table, $f_{j}=\sum_{d \in\{x, y, z\}} k_{d}\left|B_{d}^{o p t}-B_{j d}\right|$ refers to the displacement of the barycenter of bin $j$ with respect to point $(5,5,0)$, while $f_{d}=k_{d} \sum_{j \in\{1,2,3\}}\left|B_{d}^{o p t}-B_{j d}\right|$ refers to the sum, over all used bins, of the displacement in direction $d$.

185 The total displacement from the ideal barycenter is $\sum_{j \in\{1,2,3\}} f_{j}=\sum_{d \in\{x, y, z\}} f_{d}=$ 
12.713. This value is mainly given by the $z^{\text {th }}$ coordinate, plus a small unbalance of the second bin in direction $y$.

\begin{tabular}{|c|c|c|c|c|c|c|c|c|c|c|c|c|}
\hline bin & \multicolumn{7}{|c|}{ items } & & $B_{x}$ & $B_{y}$ & $B_{z}$ & $f_{j}$ \\
\hline 1 & $\{1$ & 2 & 3 & 5 & 13 & 14 & $16\}$ & & 5.000 & 5.000 & 4.520 & 4.520 \\
\hline 2 & $\{4$ & 7 & $11\}$ & & & & & & 5.000 & 5.093 & 4.333 & 4.426 \\
\hline 3 & $\{6$ & 8 & 9 & 10 & 12 & $15\}$ & & & 5.000 & 5.000 & 3.766 & 3.766 \\
\hline & & & & & & & & $f_{d}$ & 0.000 & 0.093 & 12.620 & 12.713 \\
\hline
\end{tabular}

Table 2: Items allocation and barycenter with sequential packing and balancing phases.

The integrated model arranges boxes in a different manner inside the three bins with a resulting better load balancing. Table 3 shows the new total displacement from the ideal location which is now equal to 11.332, an improvement of more than $10 \%$.

\begin{tabular}{|c|c|c|c|c|c|c|c|c|c|c|c|}
\hline bin & \multicolumn{6}{|c|}{ items } & & $B_{x}$ & $B_{y}$ & $B_{z}$ & $f_{j}$ \\
\hline 1 & $\{1$ & 8 & 10 & 12 & 15 & $16\}$ & & 5.000 & 5.000 & 3.716 & 3.716 \\
\hline 2 & $\{2$ & 3 & 5 & $14\}$ & & & & 5.000 & 5.000 & 3.341 & 3.341 \\
\hline 3 & $\{4$ & 6 & 7 & 9 & 11 & $13\}$ & & 5.000 & 5.000 & 4.265 & 4.265 \\
\hline & & & & & & & $f_{d}$ & 0.000 & 0.000 & 11.332 & 11.332 \\
\hline
\end{tabular}

Table 3: Items allocation and barycenter with integrated packing and balancing.

On the other hand, as expected, the computational time for the latter approach increases. Several minutes are needed to solve this 16-item example, compared to a few seconds for the entire sequential approach (GAMS and CPLEX 12.6.1 have been used for these experiments).

In general, very long running times occur even for small instance with only 20 items or less, thus heuristic algorithms need to be developed. The sequential approach can be used as a heuristic, but this choice may also be too complex to be solved if the size of the problem grows. Indeed, neither the MBP is able 
to solve bin-packing instances with more than a few dozens items, nor can the SBL manage situations where many items fall inside a single bin.

Many heuristics for obtaining the initial packing can be applied, e.g. the multidimensional extension of the constructive Extreme Point-based heuristics of [ $[$, but the load balancing component is poorly explored in the literature and will be the topic of the next chapter.

\section{A multi-level local search heuristic for the LB-MBP}

This section is concerned with the development of a heuristic algorithm for the

LB-MBP. The approach makes extensive use of the graph-theoretical characterization of a packing introduced by Fekete and Schepers 4, which allows us to exploit nice algorithmic properties of particular families of graphs, and to deal at the same time with classes of packings which share a certain combinatorial structure.

Intuitively, considering a set of boxes inside a bin and a specific coordinate axis, e.g. $x$, we can build a graph where:

- each node corresponds to a box

- an edge $(u, v)$ is inserted in the graph if the projections of boxes $u$ and $v$ on the $x$-axis overlap.

Figure 1 shows the construction of the interval graphs arising from a simple 2D packing.

The graph characterization of packings and its advantages are formally recalled in the following. For simplicity, notation will be slightly different.

\subsection{The graph characterization of packings}

Consider a set of $n D$-dimensional parallelepipeds $V$, the bin size $W=\left(W_{d}\right)_{d}$, and define the size function $w$ :

$$
w: V \longrightarrow \mathbb{R}^{+D}, \quad v \longmapsto w(v)=\left(w_{d}(v)\right)_{d}
$$




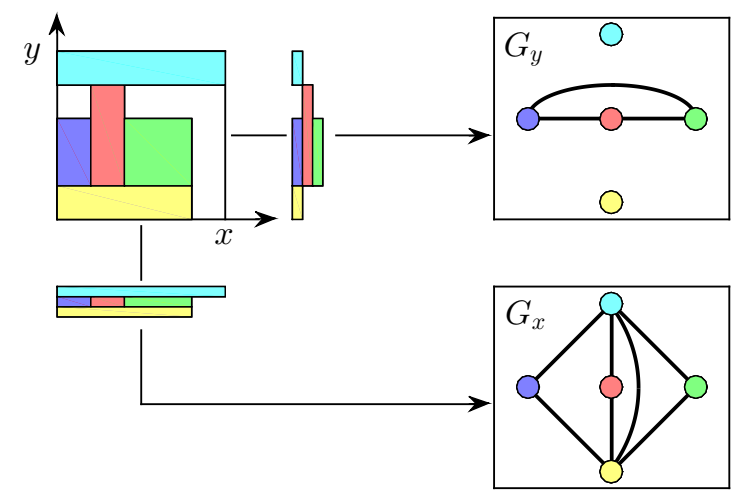

Figure 1: Interval graphs $G_{x}$ and $G_{y}$ arising from a simple 2D packing.

where $w_{d}(v)$ represents the length of box $v$ in direction $d$. Then, introduce a mapping $p: V \longrightarrow \mathbb{R}_{0}^{+D}$ which associates each box to the coordinates of its corner closest to the origin $p(v)=\left(p_{d}(v)\right)_{d}$. In this way each box position is uniquely identified. Moreover, for every $d \in D$ the function:

$$
I_{d}: \quad V \longrightarrow \mathbb{R}_{0}^{+} \times \mathbb{R}^{+}, \quad v \longmapsto I_{d}(v):=\left[p_{d}(v), p_{d}(v)+w_{d}(v)\right)
$$

maps a box $v$ onto its projection on coordinate $d$, which is an interval considered left-closed and right-open. At this point we can define a feasible packing of $(V, w, W)$ as a function $p: V \longrightarrow \mathbb{R}_{0}^{+D}$ satisfying the two conditions:

$$
\begin{array}{r}
\forall v \in V, \forall d \in D: p_{d}(v)+w_{d}(v) \leq W_{d} \\
\forall u, v \in V, u \neq v, \exists d \in D: I_{d}(u) \cap I_{d}(v)=\emptyset
\end{array}
$$

It is easy to realize that the first condition means that no box exceeds the boundaries of the bin, while the second imposes that no pair of boxes overlaps. Now, looking at the projections of the $n$ boxes onto the coordinate axes, each coordinate induces the undirected graph $G_{d}=\left(V, E_{d}\right)$ defined as:

$$
(u, v) \in E_{d} \Longleftrightarrow I_{d}(u) \cap I_{d}(v) \neq \emptyset
$$

meaning that two items are adjacent in $G_{d}$ if and only if their $d^{\text {th }}$ projections overlap. The graphs $G_{d}$ are called interval graphs, indeed, by construction, they 
are built from the intersection of intervals on the real line. We know from the non-overlapping condition that an edge $(u, v)$ cannot lie at the same time in $G_{d}$ for all $d \in D$. Furthermore, for all $G_{d}$ a stable set $S=\left\{v_{1}, \ldots, v_{k}\right\}$, i.e. a set of pairwise nonadjacent nodes, fulfills:

$$
\sum_{j=1}^{k} w_{d}\left(v_{j}\right) \leq W_{d}
$$

meaning that boxes aligned on the axis $d$ must have total length not greater than $W_{d}$. We call such a stable set $d$-feasible and sum up the previous observations with a theorem.

Theorem 1. For any feasible packing, the induced graphs $G_{d}=\left(V, E_{d}\right), d \in D$, have the following properties:

$$
\begin{array}{ll}
P_{1}: & G_{d} \text { is an interval graph } \\
P_{2}: & \text { Each stable set of } G_{d} \text { is d-feasible } \\
P_{3}: & \cap_{d} E_{d}=\emptyset .
\end{array}
$$

Fekete and Schepers showed that the reverse implication holds too and $P_{1}, P_{2}$, $P_{3}$ are sufficient to determine a feasible packing, but a few more concepts must be recalled first.

An undirected graph $G=(V, E)$ is said to be a comparability graph, or transitively orientable graph, if there exists an orientation $\Phi$ of $E$ which fulfills:

$$
(a, b) \in \Phi \wedge(b, c) \in \Phi \Longrightarrow(a, c) \in \Phi
$$

Such an orientation $\Phi$ is called a transitive orientation of $E$. Denoting $\bar{G}_{d}=$ $\left(V, \bar{E}_{d}\right)$ the complement graph of $G_{d}=\left(V, E_{d}\right)$, we know that if $G_{d}$ arises from a packing $p$, then $\bar{G}_{d}$ will be a comparability graph. Indeed, an edge in $\bar{G}_{d}$ corresponds to two boxes with non-overlapping $d^{\text {th }}$ projection; hence we can easily construct a transitive orientation $\Phi_{d}$ of $\bar{E}_{d}$ by defining:

$$
(u, v) \in \Phi_{d} \Longleftrightarrow(u, v) \in \bar{E}_{d} \wedge p_{d}(u)<p_{d}(v)
$$


i.e. projections of boxes $u$ and $v$ on axis $d$ do not overlap, and the coordinate of $u$ is smaller than $v$. More generally, we deduce that the complement graph of any interval graph is a comparability graph. The reverse implication does not hold as there exist some graphs which are not interval graphs and whose complement is transitively orientable.

Finally, a family $E=\left(E_{d}\right)_{d}$ of edge sets for a vertex set $V$ is called a packing class for $(V, w, W)$ if it satisfies the three properties $P_{1}, P_{2}, P_{3}$ and, given the transitive orientations $\Phi_{d}$ of $\bar{G}_{d}$ for $d \in D$, we call the set $\Phi=\left(\Phi_{d}\right)_{d}$ a transitive orientation of the packing class $E$.

We can now state a second important result showing that any transitive orientation $\Phi$ of $E$ induces a packing $p^{\Phi}$ and provides a method to find it by construction.

Theorem 2. For a transitive orientation $\Phi$ of a packing class $E$, the function $p^{\Phi}: V \longrightarrow \mathbb{R}_{0}^{+D}$ defined as:

$$
p_{d}^{\Phi}(v):= \begin{cases}0 & \text { if } \nexists u \in V:(u, v) \in \Phi_{d} \\ \max \left\{p_{d}^{\Phi}(u)+w_{d}(u) \mid(u, v) \in \Phi_{d}\right\} & \text { otherwise }\end{cases}
$$

for $v \in V$ and $d \in D$, represents a feasible packing.

Theorem 2 constitutes the reverse implication of Theorem 1 and motivates the graph characterization of packings. See 4 , for a proof.

By construction, the packing $p^{\Phi}$ introduced in Theorem 2 is lower gapless in every direction $d \in D$, i.e. no item can be shifted to a position with lower coordinates in some direction. Formally, a packing $p$ for $(V, w, W)$ is said to be lower gapless in direction $d$ if for all $v \in V$, either $v$ has a zero lower $d$ coordinate, $p_{d}(v)=0$, or it touches in direction $d$ another box before it:

$$
\exists u \in V: p_{d}(v)=p_{d}(u)+w_{d}(u) \wedge \forall d^{\prime} \neq d: I_{d^{\prime}}(u) \cap I_{d^{\prime}}(v) \neq \emptyset .
$$

Analogously, we say a packing $p$ for $(V, w, W)$ to be upper gapless in direction $d$ if for all $v \in V$, either $v$ touches the upper wall of the bin, $p_{d}(v)+w_{d}(v)=W_{d}$, 
or it touches in direction $d$ another box after it:

$$
\exists u \in V: p_{d}(v)+w_{d}(v)=p_{d}(u) \wedge \forall d^{\prime} \neq d: I_{d^{\prime}}(u) \cap I_{d^{\prime}}(v) \neq \emptyset
$$

and we can define the upper gapless packing $q^{\Phi}$ induced by a transitive orientation $\Phi$ by:

$$
q_{d}^{\Phi}(v):= \begin{cases}W_{d}-w_{d}(v) & \text { if } \nexists u \in V:(v, u) \in \Phi_{d} \\ \min \left\{q_{d}^{\Phi}(u)-w_{d}(v) \mid(v, u) \in \Phi_{d}\right\} & \text { otherwise. }\end{cases}
$$

To simplify notation, $p$ and $q$ will denote the two lower and upper gapless packings induced by a transitive orientation $\Phi$, and $p_{d}, q_{d}: V \longrightarrow \mathbb{R}_{0}^{+}$their components. The single components of $p$ and $q$ are defined mutually independent, thus having $\Phi$ we can generate packings which are mixed lower and upper gapless in the various directions, i.e. $\left(p_{d_{1}}, q_{d_{2}}\right), d_{1} \in D_{1}, d_{2} \in D_{2}$, with $\left\{D_{1}, D_{2}\right\}$ a partition of $D$.

Introducing the gapless packings induced by a transitive orientation is important when handling the load-balancing, as will be illustrated in the next section.

\subsection{Basic balancing operations}

Going back to the load-balancing problem, assume a bin-packing solution is available and focus on a single bin. We want to rearrange items inside the bin in order to make the barycenter of the bin as close as possible to the ideal point $B^{\text {opt }}$. Given the packing $p$, it is possible to apply an overturning operation $\bar{p}$ :

$$
\bar{p}: V \longrightarrow \mathbb{R}_{0}^{+D}, \quad v \longmapsto \bar{p}(v):=W-p(v)-w(v)
$$

representing the symmetric packing of $p$ w.r.t. the $D$ hyperplanes which halve the bin at $W_{d} / 2$. Note that the feasibility of the solution is obviously preserved together with the gapless property (now upper instead of lower), and the operation can be restricted to a single direction $d \in D$, leaving the remaining coordinates unchanged. The same operation can be done with $q$, obtaining $\bar{q}$.

${ }_{255}$ Consider now the packing class $E$ associated with the bin and its transitive orientation $\Phi$. We can reallocate the boxes using four induced gapless packings, 
$p, q, \bar{p}, \bar{q}$. Moreover, again, we can create a packing with mixed components $p_{d}$, $q_{d}, \bar{p}_{d}, \bar{q}_{d}$ as they act independently in the definition of $p, q, \bar{p}, \bar{q}$. Thus, a transitive orientation $\Phi$ induces $4^{D}$ gapless packings, each of them with a different barycenter.

Let us focus on a single direction $d$ and denote with $B_{d}^{p}, B_{d}^{q}, B_{d}^{\bar{p}}$, $B_{d}^{\bar{q}}$ the $d^{\text {th }}$ coordinate of the barycenter obtained with $p_{d}, q_{d}, \bar{p}_{d}, \bar{q}_{d}$ respectively. We know that $B_{d}^{p} \leq B_{d}^{q}$ and $B_{d}^{\bar{q}} \leq B_{d}^{\bar{p}}$.

Two cases are possible:

In the first case, as shown in Figure 2, the best we can do to balance the packing is simply choosing the point among $B_{d}^{p}, B_{d}^{q}, B_{d}^{\bar{p}}, B_{d}^{\bar{q}}$ closest to $B_{d}^{\text {opt }}$ and the corresponding packing component.

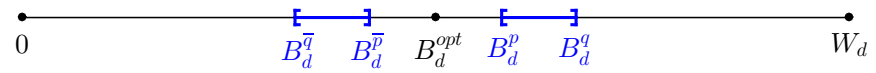

Figure 2: $d^{\text {th }}$ coordinate of the barycenter of the four gapless packings. In the example $B_{d}^{o p t} \notin\left[B_{d}^{p}, B_{d}^{q}\right] \cup\left[B_{d}^{\bar{q}}, B_{d}^{\bar{p}}\right]$ and the packing component $p_{d}$ is chosen.

In the second case, we will show that a packing with the optimal barycenter coordinate $B_{d}^{o p t}$ can be determined. Assume WLOG that $B_{d}^{o p t} \in\left[B_{d}^{p}, B_{d}^{q}\right]$ and investigate in dept the relationship between $p_{d}$ and $q_{d}$. Starting with $p_{d}$, we can take the box which has been last provided with its coordinates by $p_{d}$, and push it towards the upper wall of the bin until the item touches it. Then take the second to last box to be assigned coordinates, and push it towards the upper coordinates until it touches the wall of the bin or the previous box, and continue this way until all items have been considered. What we get at the end is a feasible packing, upper gapless in direction $d$, which coincides with $q_{d}$. In other words, it is possible to go from $p_{d}$ to $q_{d}$ (or from $q_{d}$ to $p_{d}$ ) shifting items in direction $d$ toward higher (or lower) coordinates and following a specific order. Each time we move a box the barycenter moves. Having $B_{d}^{o p t} \in\left[B_{d}^{p}, B_{d}^{q}\right]$, there 
must be one item, say $v$, whose shift makes the barycenter crossing the optimal $d^{\text {th }}$ coordinate $B_{d}^{o p t}$, i.e. it moves from $B_{d}^{1}$ to $B_{d}^{2}$ with $B_{d}^{1} \leq B_{d}^{o p t} \leq B_{d}^{2}$. Then, item $v$ can be provided with a $d^{t h}$ coordinate such that the barycenter is exactly $B_{d}^{o p t}$, simply using the proportion:

$$
v \longmapsto p_{d}(v)+\left(B_{d}^{2}-B_{d}^{1}\right)^{-1}\left(q_{d}(v)-p_{d}(v)\right)\left(B_{d}^{o p t}-B_{d}^{1}\right)
$$

Notice that if $B_{d}^{\text {opt }}=\frac{1}{2} W_{d}$, then for symmetry reasons the overturning is redundant.

To summarize, given a packing class with a transitive orientation, we can make the barycenter closer to the ideal point by taking simple choices involving the gapless packings induced by the orientation and performing basic operations as moving iteratively boxes along one direction and overturning the packing. This is likely the best we can do right now with a specific transitive orientation, but we will soon manage to find more transitive orientations of a packing class.

\subsection{Local search at transitive orientation level}

The goal of this section is to find a transitive orientation of a packing class which leads, after performing the basic operations previously mentioned, to a well-balanced packing. First, it is useful to determine how many transitive orientations a comparability graph has and how to construct them. Terminology and results of graph theory are needed and in the following only a few important points will be mentioned. For an extensive background on the topic refer to Golumbic 25].

It can be proven that the edges of an undirected graph $G=(V, E)$ can be uniquely partitioned into substructures $M_{1}, \ldots, M_{k}$, called maximal multiplexes, which act independently to transitive orientations. We say that $E=M_{1}+\cdots+$ $M_{k}$ is the $M$-decomposition of the graph $G$.

To identify a maximal multiplex $M_{i}$, we first need to find a maximal simplex $S_{i}$, i.e. a complete subgraph with certain properties which can be built up by local search from a single edge, and then make it grow using a particular equivalent relation that forces adjacent edges to be part of the same structure. 
A simplex $S_{i}$ with $r+1$ vertices, or with rank $r$, has $(r+1)$ ! transitive orientations induced by any linear ordering of its vertices. More formally, assume WLOG its vertices to be $0,1, \ldots, r_{i}$, consider any position function, i.e. a bijection $\operatorname{pos}_{i}:\left\{0,1, \ldots, r_{i}\right\} \longrightarrow\left\{0,1, \ldots, r_{i}\right\}$, and for all $u, v \in\left\{0,1, \ldots, r_{i}\right\}, u \neq v$, state that:

$$
(u, v) \in \Phi \Longleftrightarrow \operatorname{pos}_{i}(u)<\operatorname{pos}_{i}(v)
$$

For instance, for each maximal simplex $S_{i}$ we can choose the identity function on $\left\{0,1, \ldots, r_{i}\right\}$, so that edge $\left(r_{i}, r_{j}\right) \in \Phi$ if and only if $i<j$. Such orientation $\Phi$ is easily proven to be transitive. Now, we inductively extend the orientation of each maximal simplex $S_{i}$ to its maximal multiplex $M_{i}$, making $\Phi$ a transitive orientation of the whole graph. A theorem in [25] ensures that a transitive orientation of a simplex uniquely extends to a transitive orientation of the multiplex generated from it (except when the multiplex itself is not transitively orientable). Conversely, a transitive orientation of a multiplex uniquely restricts to a transitive orientation of any simplex contained in it. Finally, denoting with $t(G)$ the number of transitive orientations of a graph $G$, we recall the following central result.

Theorem 3. Let $G=(V, E)$ be an undirected graph, and consider its $M$ decomposition $E=M_{1}+\cdots+M_{k}$.

(i) If $\Phi$ is a transitive orientation of $G$, then $\Phi \cap M_{i}$ is a transitive orientation of $M_{i}$

(ii) If $\Phi_{1}, \ldots, \Phi_{k}$ are transitive orientations of $M_{1}, \ldots, M_{k}$ respectively, then $\Phi_{1}+\cdots+\Phi_{k}$ is a transitive orientation of $G$

(iii) $t(G)=t\left(M_{1}\right) \cdot t\left(M_{2}\right) \cdots t\left(M_{k}\right)$

(iv) If $G$ is a comparability graph and rank $M_{i}=r_{i}$, then $t(G)=\prod_{i=1}^{k}\left(r_{i}+1\right)$ !.

We now have a procedure to determine all transitive orientations of a comparability graph. First decompose it into maximal simplices $S_{1}, \ldots, S_{k}$ and maximal 300 multiplexes $M_{1}, \ldots, M_{k}$. Any linear ordering of the vertices of a simplex $S_{i}$ induces a transitive orientation $\Phi_{i}$ of $S_{i}$, which is then extended to a transitive 
orientation to $M_{i}$. The union of the $\Phi_{i}$ over all maximal multiplexes constitutes a transitive orientation for the entire graph.

The next step is to move to the space of transitive orientations in order to find a well-balanced packing. The analysis can be done for each coordinate independently of the others, so we focus on a single $d \in D$.

Given two transitive orientations $\Phi, \Psi$ of $\bar{G}_{d}$, we evaluate them stating that $\Phi$ is preferable to $\Psi$ if $f_{d}(\Phi) \leq f_{d}(\Psi)$, where $f_{d}(\Phi)=\left|B_{d}^{o p t}-B_{d}(\Phi)\right|$ and $B_{d}(\Phi)$ is the best barycenter we can obtain from $\Phi$ performing the basic balancing operations (overturning, shifting items etc.) described in Section 3.2.

The number of transitive orientations of $\bar{G}_{d}=\left(V, \bar{E}_{d}\right)$ is a product of factorials, $t\left(\bar{G}_{d}\right)=\prod_{i=1}^{k}\left(r_{i}+1\right)$ ! where $k$ is the number of maximal simplices and $r_{i}$ is the rank of simplex $i$. If $\bar{E}_{d}$ itself is a clique, then $t\left(\bar{G}_{d}\right)$ assumes its maximum possible value $t(G)=|V|$ !. Such a number could potentially be huge. As a consequence, instead of evaluating all transitive orientations, we will only explore a polynomial subset of orientations.

In order to move from $\Phi$ to another transitive orientation $\Psi$ which is "close" in some sense to $\Phi$, we can modify the position function of one simplex, let's say $S_{i}$, inverting the position of a pair of nodes $\operatorname{pos}_{i}(u) \longleftrightarrow \operatorname{pos}_{i}(v)$, as illustrated in Figure 3 .
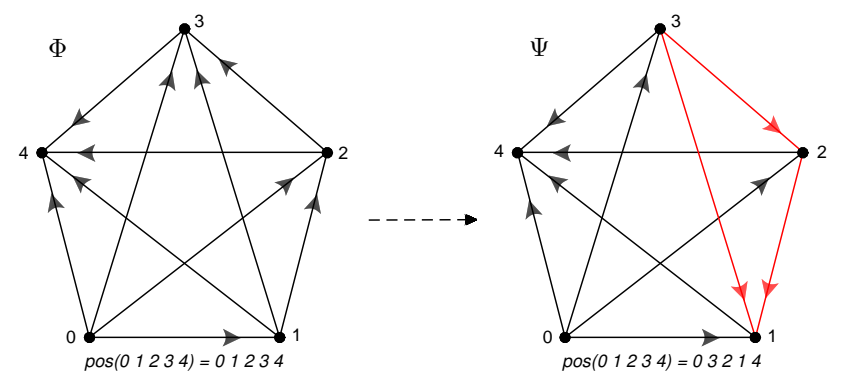

Figure 3: Swapping the position function of nodes 1 and 3 in a simplex of rank 4 . The new orientation differs by the red edges.

This kind of swap can be performed for each pair of nodes of $S_{i}$, every time reorienting the simplex and extending the new orientation to its maximal mul- 
tiplex $M_{i}$ to have a transitive orientation of the whole graph (in the meanwhile, the other multiplexes with their orientation are left unchanged). If simplex $S_{i}$ has rank $r_{i}$, then the number of couples of nodes is $r_{i}\left(r_{i}+1\right) / 2$, so we are considering a quadratic subset of the total factorial number of transitive orientations of simplex $S_{i}$.

\subsubsection{Balancing using transitive orientations: the algorithm}

The algorithm is designed as a hill-climber heuristic and is here outlined. The dimensions $d \in D$ are considered separately one by one. First, decompose $\bar{G}_{d}$ into maximal simplices and maximal multiplexes. Take the first simplex and evaluate all the exchanges of vertice pairs w.r.t. the initial position function. Save the best barycenter solution and its relative position function, then move to the next simplex. The process is repeated until it has considered all simplices without having found any improving solution, or when a maximum number of iterations is reached. Finally, repeat the process for the other coordinates $d \in D$.

The algorithm is classified as a local search method since we move from one orientation $\Phi$ to a nearby one $\Psi$ obtained by swapping the position of two vertices in a simplex. The neighborhood of $\Phi$ is defined as the set of transitive orientations generated from $\Phi$ by a swap in the position function of two nodes in the same simplex. In particular, since we create a sequence of improving solutions

$$
f_{d}\left(\Phi_{0}\right)>f_{d}\left(\Phi_{1}\right)>\cdots>f_{d}\left(\Phi_{k}\right)
$$

the algorithm can be identified as a hill-climbing search. Moreover, we explore the whole quadratic neighborhood of a simplex $S_{i}$, pick up the best solution, and then go to $S_{i+1}$. Thus, the algorithm is a best improvement search. It could equally well have been defined as a first improvement search, indeed, in several local search methods a first improvement strategy drastically reduces the running time without degrading the solution quality. However, in our case 335 the best improvement search performed slightly better than first improvement. 


\subsubsection{Balancing using transitive orientations: an example}

To clarify the previous concepts, consider the $2 \mathrm{D}$ instance with 5 rectangles described in Table 4. The bin is a square with lengths 5 and the optimal barycenter is its geometric center $B_{\text {opt }}=(2.5,2.5)$.

\begin{tabular}{cccccc}
\hline item & 1 & 2 & 3 & 4 & 5 \\
\hline$w_{x}$ & 4 & 1 & 1 & 2 & 5 \\
$w_{y}$ & 1 & 3 & 2 & 2 & 1 \\
$\rho$ & 1 & 5 & 1 & 1 & 3 \\
\hline
\end{tabular}

Table 4: 2D 5-item instance used to illustrate the load-balancing with transitive orientations.

340 Given a packed bin, we extract the two complementary graphs $\bar{G}_{x}, \bar{G}_{y}$ with the initial orientations $\Phi_{x}, \Phi_{y}$. After the basic balancing operations have been performed, we obtaining the barycenter of $B=(2.32,2.97)$. The situation is shown in the top of Figure 4

Examining $\bar{G}_{x}$, a new transitive orientation $\Psi_{x}$ is found. Using $\Psi_{x}$ we are able to determine a different packing whose barycenter has a $x$ coordinate $B_{x}=2.5$. The situation concerning the $y$ direction has not changed, so the new location is $B=(2.5,2.97)$.

Finally, take $\bar{G}_{y}$ into account. The item located at the top of the packing is heavy compared to the others $(\rho=3)$ and we are able to find a new transitive orientation, $\Psi_{y}$, such that this item will have a new lower coordinate. Using $\Psi_{x}$ and $\Psi_{y}$, the barycenter reaches $B=(2.5,2.5)$, so the packing is perfectly balanced in both coordinates, and the algorithm stops.

To summarize, a local search-based algorithm has been developed. It is able to move among the transitive orientations of a packing class and looks for the most balanced packing. Since there can be a factorial number of transitive orientations, only a polynomial subset of them is explored.

\subsection{Local search at graph level}

It may happen that the set of transitive orientations of a graph is very limited and the local search described above is consequently weak. Hence, another level 


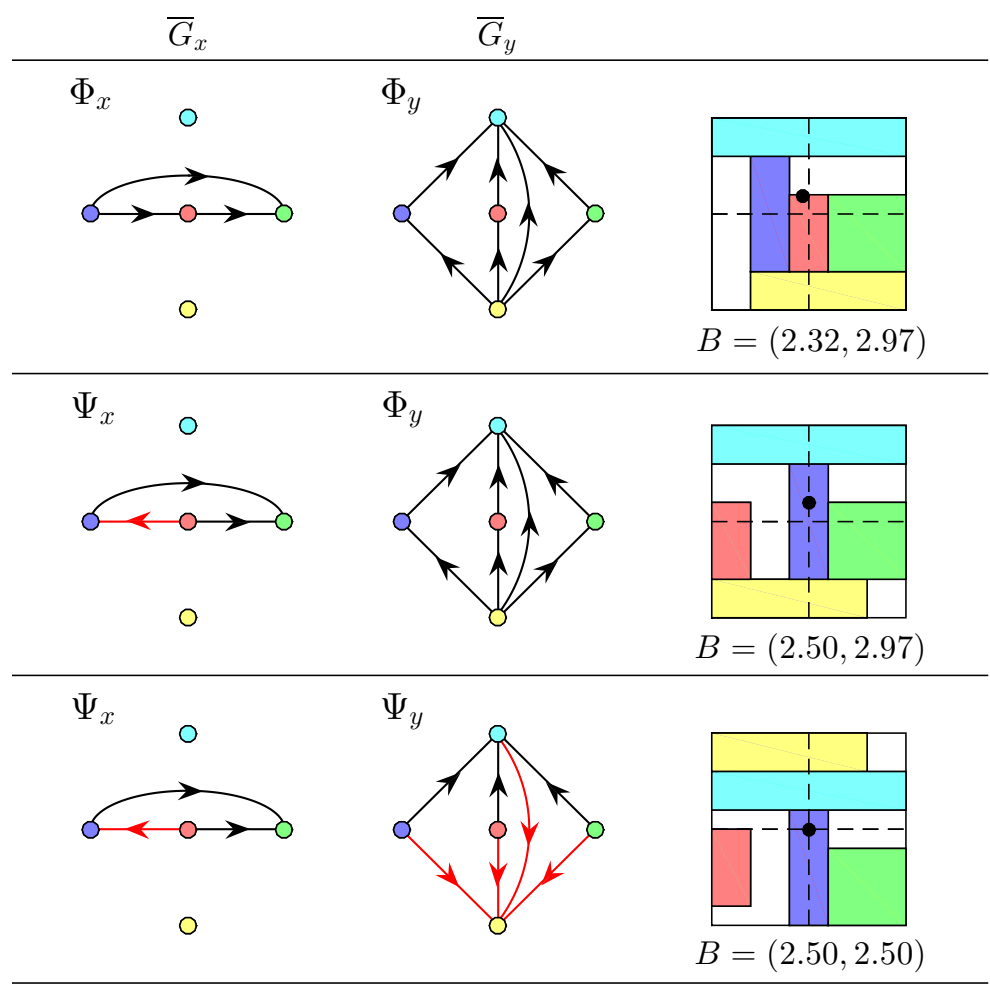

Figure 4: Load balance of a two-dimensional bin. For each of the three steps (top, middle, bottom) the two complement graphs $\bar{G}_{x}, \bar{G}_{y}$, their transitive orientations and the packing resulting from the balancing operations of section 3.2 are shown. The red arrows indicate where the orientation has changed.

can be added on top of the algorithm, consisting in modifying the packing class itself. In the previous sections the packing class has never touched, we only "played" with its orientations.

Here again we look at the single bin, but we change the structure of the $D$ graphs $G_{d}=\left(V, E_{d}\right)$ adding or removing edges, with the purpose of moving away from possible limited groups of packings sharing a similar combinatorial structure given by the packing class. The main idea is that whenever we obtain a new packing class, we perform the local search among transitive orientations. To do this, we have to deal with two main difficulties: 
- How to modify a packing class in a clever way?

To modify the packing class we follow the approach of Crainic et al. 9]. The authors proposed a neighborhood for a packing class, based on what they call overlapping rules, and use it in the inner heuristic of a two-level tabu-search for the 3D Bin-Packing Problem.

Basically, for each $d$ and couple of items $u \neq v$ in the bin, either $(u, v) \in E_{d}$ if $u$ and $v$ overlap in direction $d$, or $(u, v) \notin E_{d}$ if they do not. In the first case, items can be made non-overlapping by removing edge $(u, v)$ form $E_{d}$, whereas in the second case they are made overlapping by adding $(u, v)$ to $E_{d}$, but avoiding the same edge being present in $E_{d}$ for all $d \in D$.

In order to increase the probability for the new set of graphs to be a packing class, some heuristic rules are used when adding or removing an edge. In particular, when $(u, v)$ is added to $E_{d}$, a new edge set $E_{d}^{\prime}$ is built following the so called add rule:

$$
\begin{aligned}
E_{d}^{\prime} & =E_{d} \cup\{(u, v)\} \cup\left\{(u, s): p_{d}(s) \leq p_{d}(v) \wedge p_{d}(s)+w_{d}(s) \geq p_{d}(v)+w_{d}(v)\right\} \\
& =E_{d} \cup\{(u, v)\} \cup\left\{(u, s): I_{d}(v) \subseteq I_{d}(s)\right\}
\end{aligned}
$$

meaning that if $u$ and $v$ overlap, then $u$ and $s$ must overlap too for each $s$ such that $I_{d}(v) \subseteq I_{d}(s)$. Similarly, if $(u, v)$ is removed from $E_{d}$, then we construct a new edge set $E_{d}^{\prime}$ following the remove rule:

$$
\begin{aligned}
E_{d}^{\prime} & =E_{d} \backslash\left\{\{(u, v)\} \cup\left\{(u, s): p_{d}(s) \geq p_{d}(v) \wedge p_{d}(s)+w_{d}(s) \leq p_{d}(v)+w_{d}(v)\right\}\right\} \\
& =E_{d} \backslash\left\{\{(u, v)\} \cup\left\{(u, s): I_{d}(v) \supseteq I_{d}(s)\right\}\right\}
\end{aligned}
$$

where edges $(u, s)$ are also removed from $E_{d}$ if $I_{d}(v) \supseteq I_{d}(s)$.

Once we have a new set of graphs $G_{d}$, we need to check if they correspond to a packing class, i.e. whether conditions $P_{1}, P_{2}$ and $P_{3}$ are fulfilled. From graph theory we know that $G$ is an interval graph if and only if $G$ does not 375 contain any $C 4$ chordless cycle, i.e. a chordless cycle with four nodes, and $\bar{G}$ is 
a comparability graph. Thus, from a computational point of view, the graphs $G_{d}=\left(V, E_{d}\right), d \in D$ represent a packing class if:

1. $\forall d, G_{d}$ does not contain any $C 4$ chordless cycle

2. $\forall d$, there exists a transitive orientation of $\bar{G}_{d}$ obtain different partial solutions to the bin-packing problem and, when a single bin is still used, we balance it and keep the new packing in case of a balancing improvement. This diversification phase has been introduced since the local 

packing class.

Secondly, similar operations are performed with n-tuples of bins, as described below:

1. Scores are assigned to bins reflecting the current imbalance or the possi-

search in graphs alone may not be able to explore regions far from the initial bility of balancing improvement. In particular, we defined two scores per bin: $S_{1, i}=f_{i}^{2}$ and $S_{2, i}=1-V_{i}$, where $f_{i}$ is the imbalance of bin $i$ and $V_{i}$ is the percentage of volume filling in bin $i$.

2. $k$ bins are randomly chosen with the operation usually called roulette wheel selection, i.e. using probabilities proportional to the scores. In our implementation, bin 1 out of $k$ is chosen with score $S_{1}$, and the remaining $k-1$ bins with score $S_{2}$.

3. The items in the $k$ selected bins are merged and a randomized constructive heuristic is used to recombine them.

4. If $k$ bins are still used in the new solution, each of them is balanced. When the imbalance sum over the $k$ repacked bins decreases, the new partial packing is saved and the scores updated.

Before adopting such methodology, we need to choose the number $k$ of bins to repack.

We denote a $k$-neighborhood as the set of bin-packing solutions obtained by repacking at least $k$ bins (successfully, without increasing the number of used bins), i.e. solutions which differ in the packing of at most $k$ bins with respect to the initial packing. Since the size of $k$-neighborhoods increases exponentially with $k$, we start considering couples $(k=2)$ as they are faster to explore. If no balancing improvements are made in a predetermined number of iterations, the search is extended to triples $(k=3)$ and so on until a maximum level of $k$ (e.g. $k=8)$ is explored without any improving solution.

We also allow the search to go back to prior levels. In particular, after level $k>2$ is explored, we move back to level 2 if any improvement was made at level $k$, otherwise we proceed to next level $k+1$. 
This approach is called variable-depth neighborhood search (VDNS) as the size of the $k$-neighborhood is not fixed but dynamically adjusts itself during the search. See Ahuja et al. 26] or Pisinger and Ropke 27] for a detailed discussion on VDNS.

Note that the $\mathscr{L}_{1}$-norm was necessary to build the MILP models in Chapter

440 2. For the heuristic algorithm any other measure of imbalance can be chosen instead, e.g. the Euclidean norm.

\section{Computational results}

The heuristic algorithm for the LB-MBP is now applied to several test cases. For the experiments we will focus on the three-dimensional problem, but everything discussed so far applies to any dimension.

\subsection{Finding the initial 3D bin-packing solution}

We make use of a variant of the Extreme Point First Fit Decreasing heuristic (EPFFD) 8], outlined here.

Boxes are initially sorted by non-increasing volume. Two consecutive boxes may have a similar volume but a very different shape, e.g tall with small base area vs. short and wide. If we swap their ordering and then apply an Extreme Point-based algorithm, the final packing will be different and, if we are lucky, we will save a bin. Thus, we start considering the "ideal" sorting by non-increasing volume and, for each couple of consecutive boxes, we exchange their ordering with a certain probability related to the ratio of their volume. The probability $p_{i}$ of swapping box $i$ and $i+1$ is defined by:

$$
p_{i}:= \begin{cases}5\left(\frac{\text { vol }_{i+1}}{\text { vol }_{i}}-0.9\right) & \text { if } \frac{\text { vol }_{i+1}}{\text { vol }_{i}}>0.9 \\ 0 & \text { if } \frac{\text { vol }_{i+1}}{\text { vol }_{i}} \leq 0.9\end{cases}
$$

Therefore, for each box $i$, we compute $p_{i}$, generate a random value $r \sim U(0,1)$ and swap $i$ with $i+1$ if $r<p_{i}$. Labels are updated immediately after each swap so that a box may shift more positions. After this operation we run the 
the best solution is saved.

We call this algorithm GRASP-Based EPFFD, or G-EPFFD, since the greedy randomized initialization reminds us of the first part of the GRASP metaheuristic, although there is no succeeding search phase. Despite the simplicity thousands of times during the VDNS.

\subsection{Load Balancing}

The multi-level local search algorithm is used to balance the initial bin-packing solution obtained from G-EPFFD on a variety of test cases summarized in Tables 4755 and 6 .

The desired barycenter location is the center of the bin $B^{o p t}=0.5\left(W_{x}, W_{y}, W_{z}\right)$ in Table 5 and the center of the base of the bin $B^{o p t}=0.5\left(W_{x}, W_{y}, 0\right)$ in Table 6. Classes 1-8 are standard instances for the 3D Bin-Packing Problem from the 
literature 3 tested with 100 and 200 items. Columns 3 and 4 contain the lower bound on the number of bins and the G-EPFFD solution respectively. Each class is then tested with three different density distributions of items:

1. same density for all items, $\rho_{i}=1$

2. small density variance, $\rho_{i} \sim U(1,2)$

3. high density variance, $\rho_{i} \sim U(1,6)$

For every test case we measured how the balancing component of the objective function (14) decreases when the different search levels are used, keeping the weights $k_{x}=k_{y}=k_{z}=1$. The first phase ( $\left.\mathrm{Ph} 1\right)$ represents the imbalance value immediately after G-EPFFD is applied, the second phase $(\mathrm{Ph} 2)$ gives the value after each bin is balanced, the last phase $(\mathrm{Ph} 3)$ indicates the final imbalance value after VDNS is performed.

To allow a comparison between different test cases, entries of Tables 5 and 6 are normalized so that they contain the mean imbalance value per individual bin, and refer to bins of edge length 100. Moreover, all entries are averages over 25 instances.

${ }_{495}$ The algorithm has been implemented in $\mathrm{C}$ and run on an Intel Core i5 with 8GB RAM. The average running time varies from a few seconds (Table 5 class 1-4) to a few minutes (Table 6, class 5).

When the optimal barycenter is the center of the bin (Table 5), a lower bound for the balancing component is trivially zero, corresponding to zero imbalance in every direction for all bins and hence leading to an easy interpretation of the results.

After each bin is individually balanced $(\mathrm{Ph} \mathrm{2})$, the objective value is greatly improved and on average more than $90 \%$ of the total initial imbalance is removed from the packing. The last balancing phase $(\mathrm{Ph} 3)$ has also a considerable effect, bringing the objective value very close to zero in most of the cases and showing once again the limits of the sequential packing and balancing approach (Ph $1+$ $\mathrm{Ph}$ 2) compared to a unified approach where the balancing potential of having 


\begin{tabular}{|c|c|c|c|c|c|c|c|c|c|c|c|c|}
\hline \multicolumn{13}{|c|}{ Foo } \\
\hline \multicolumn{4}{|c|}{$B^{o p t}=$ center of the bin } & \multicolumn{3}{|c|}{$\rho_{i}=1$} & \multicolumn{3}{|c|}{$\rho_{i} \sim U(1,2)$} & \multicolumn{3}{|c|}{$\rho_{i} \sim U(1,6)$} \\
\hline class & size & LB & bins & $\mathrm{Ph} 1$ & $\mathrm{Ph} 2$ & $\mathrm{Ph} 3$ & $\mathrm{Ph} 1$ & $\mathrm{Ph} 2$ & $\mathrm{Ph} 3$ & $\mathrm{Ph} 1$ & $\mathrm{Ph} 2$ & $\mathrm{Ph} 3$ \\
\hline 1 & 100 & 24.12 & 25.64 & 16.48 & 0.91 & 0.011 & 17.03 & 0.68 & 0.054 & 19.22 & 2.26 & 0.201 \\
\hline 2 & 100 & 24.64 & 26.12 & 16.19 & 0.85 & 0.011 & 16.69 & 0.68 & 0.042 & 19.01 & 2.28 & 0.198 \\
\hline 3 & 100 & 24.48 & 26.08 & 16.34 & 0.87 & 0.006 & 16.78 & 0.66 & 0.030 & 19.16 & 2.21 & 0.245 \\
\hline 4 & 100 & 57.44 & 60.60 & 31.74 & 0.58 & 0.011 & 30.64 & 0.29 & 0.030 & 30.91 & 0.93 & 0.090 \\
\hline 5 & 100 & 13.60 & 14.60 & 15.83 & 0.92 & 0.003 & 15.87 & 0.71 & 0.068 & 17.65 & 2.70 & 0.425 \\
\hline 6 & 100 & 18.20 & 20.08 & 9.83 & 1.45 & 0.190 & 10.30 & 1.70 & 0.396 & 13.49 & 4.54 & 1.210 \\
\hline 7 & 100 & 11.12 & 12.36 & 16.89 & 1.19 & 0.012 & 16.53 & 0.79 & 0.050 & 18.37 & 3.11 & 0.559 \\
\hline 8 & 100 & 15.52 & 17.08 & 15.66 & 0.78 & 0.008 & 15.34 & 0.64 & 0.056 & 17.35 & 2.63 & 0.501 \\
\hline 1 & 200 & 48.84 & 51.16 & 15.05 & 0.79 & 0.007 & 15.74 & 0.82 & 0.032 & 18.36 & 2.57 & 0.208 \\
\hline 2 & 200 & 48.48 & 50.80 & 14.81 & 0.77 & 0.006 & 15.57 & 0.85 & 0.044 & 18.24 & 2.58 & 0.217 \\
\hline 3 & 200 & 49.24 & 51.24 & 14.91 & 0.77 & 0.005 & 15.68 & 0.83 & 0.036 & 18.29 & 2.65 & 0.262 \\
\hline 4 & 200 & 117.8 & 122.2 & 31.91 & 0.53 & 0.004 & 30.66 & 0.29 & 0.012 & 30.91 & 0.96 & 0.034 \\
\hline 5 & 200 & 25.60 & 27.36 & 12.47 & 0.75 & 0.011 & 13.06 & 0.89 & 0.147 & 14.90 & 3.23 & 0.801 \\
\hline 6 & 200 & 35.84 & 38.24 & 6.74 & 1.30 & 0.241 & 8.17 & 2.19 & 0.523 & 12.09 & 5.44 & 1.505 \\
\hline 7 & 200 & 20.36 & 22.40 & 12.72 & 1.26 & 0.057 & 12.95 & 1.15 & 0.329 & 14.89 & 3.99 & 1.567 \\
\hline 8 & 200 & 29.40 & 32.04 & 12.75 & 0.70 & 0.016 & 12.79 & 0.98 & 0.183 & 14.66 & 3.33 & 0.921 \\
\hline
\end{tabular}

Table 5: Multi-level local search heuristic for LB-MBP (3D case) when optimal barycenter is the center of the container

a large number of packing solutions with the same number of bins is exploited.

${ }_{510}$ We notice that it is easier to arrange equally dense items in the bin space so that the barycenter falls close to the center of the bin. Such a task is more difficult when some very dense item is present and the balancing is consequently slightly worse.

When the optimal barycenter is the center of the base of the container (Table 515 6), zero is still an obvious lower bound but not tight enough to be somehow useful. The bin imbalance may be zero on the $x$ and $y$ directions, but it will always be strictly positive on $z$.

It is possible to construct a new lower bound by "liquifying" the items and 


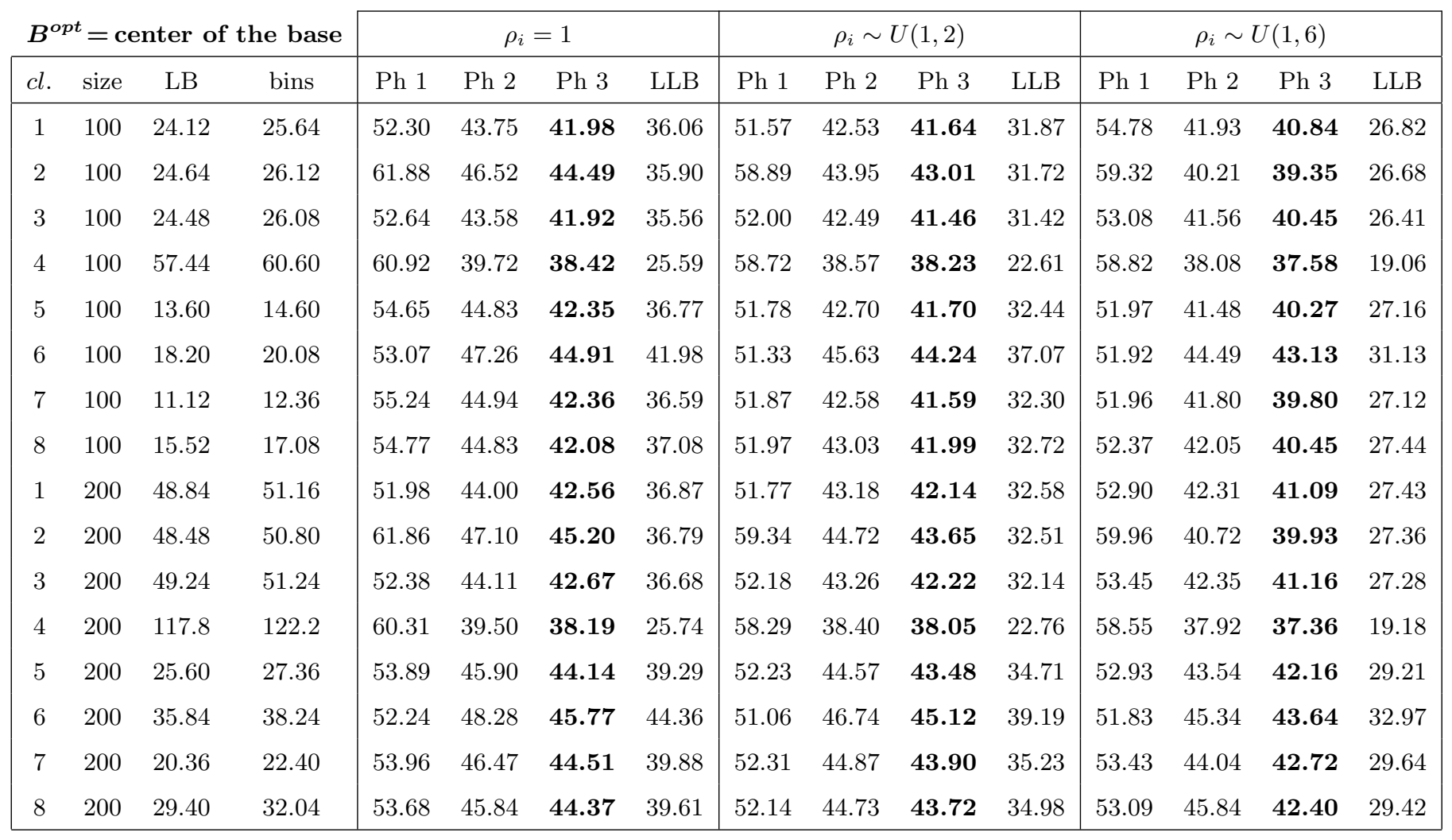

Table 6: Multi-level local search heuristic for LB-MBP (3D case) when optimal barycenter is the center of the base of the container

inserting them in the bins in a specific way. Assume the packing solution uses ${ }_{520} k$ bins and that the $n$ items are sorted by non-increasing density $\rho_{1} \geq \rho_{2} \geq$ $\cdots \geq \rho_{n}$. Take the first item and spread it on the bottom of all the $k$ bins, creating $k$ identical layers of thickness equal to $\frac{v o l_{i}}{k W_{x} W_{y}}$. Then take the second item and spread it again in every bin, creating $k$ new identical layers on top of the previous layers, and proceed this way until all items are considered. At ${ }_{525}$ the end of the process each bin will contain the same configuration of $n$ layers ordered by density, and its barycenter represents a lower bound on the average bin imbalance (see Appendix A for a formal proof). We call it the Liquid Lower Bound (LLB) and report it in Table 6.

The load balancing is again effective using the different search phases and the 
Ph 3. The gap from LLB remains quite high around 20-25\%; however, given the small gaps achieved in Table 5, we tend to believe that in Table 6 we are also not far from the optimal balancing.

LLB is clearly not very tight for a general instance since we are introducing two splittable into more bins. We suspect it is possible to construct a tighter lower bound by exploiting the unsplittable and 3D shape of items with combinatorial logic. This task, together with providing bounds for the general case when the desired barycenter is neither the center of the bin nor the center of its base, is

Finally, we notice that the balancing value is now slightly better when densities vary a lot from each other. Indeed it is possible to locate heavy items at the bottom of the bin, moving down the barycenter.

\section{Conclusions}

545 In this paper, we presented the load-balanced multi-dimensional bin-packing problem, aimed at developing an integrated model of the multi-dimensional bin packing with the load balancing of bins. The problem has only been considered in the literature in simplified versions (e.g. balancing a single bin or introducing a feasible region for the barycenter) and is here treated in its natural full

We developed a mathematical programming model able to optimally solve smaller instances of the problem, and defined a multi-level local search heuristic able to handle large instances. Main ingredients of the heuristic are the graph representation of packings, the analysis of the transitive orientations of a graph, the extreme point-based algorithms for the packing phases and the dynamical recombination of n-tuples of weakly balanced bins.

Computational experiments were promising, showing that an effective load balancing can be obtained. Moreover, despite the intrinsic complexity of the algo- 
rithm (many stages nested into each other), the running time of the heuristic is usually very low and rarely exceeds 3-5 minutes even for difficult or large instances with 200 items, making the algorithm applicable in a set of real-life logistic problems.

\section{Acknowledgments}

The authors would like to thank Prof. Marco Trubian and Dr. Fabio Colombo,

University of Milan, for the useful suggestions provided to this work.

\section{Appendix A. Liquid lower bound}

In order to prove that LLB represents a lower bound, we show that the average barycenter of $k$ containers filled with any different arrangement of liquefied items (not necessarily by layers) would be higher.

Let us call $\Omega_{i}, i=1, \ldots, k$ the $3 \mathrm{D}$ volume occupied by container $i$, and dispose the liquefied items into the containers in a different way. Assume containers are stick together and remove the separating walls, obtaining one super bin which occupies a $3 \mathrm{D}$ volume $\Omega=\cup_{i} \Omega_{i}$. It is known from fluid dynamics that the state of lowest potential energy in this super bin corresponds to the horizontal density stratification where lightest fluids are on the top, as in LLB (see for instance [28]). A lower potential energy of the system implies a lower barycenter, being

the potential energy $U=\int_{\Omega} \rho(x, y, z) g z d \Omega$ proportional to the $z^{\text {th }}$ coordinate of the barycenter $S_{z}=\frac{1}{M} \int_{\Omega} \rho(x, y, z) z d \Omega$, with $M=\int_{\Omega} \rho(x, y, z) d \Omega$ total mass.

Notice that the $z^{\text {th }}$ coordinate of the barycenter of the super bin, $S_{z}$, does not necessarily coincide with the average $z^{t h}$ coordinate of the barycenter of the $k$ bins, $B_{z}$, as shown in the example of Figure 5. Let us analyze the relationship between these two values.

$$
S_{z}=\frac{1}{M} \int_{\Omega} \rho(x, y, z) z d \Omega=\frac{\int_{\Omega} \rho(x, y, z) z d \Omega}{\int_{\Omega} \rho(x, y, z) d \Omega}=\frac{\sum_{i=1}^{k} \int_{\Omega_{i}} \rho(x, y, z) z d \Omega}{\sum_{i=1}^{k} \int_{\Omega_{i}} \rho(x, y, z) d \Omega}
$$


while:

$$
B_{z}=\frac{1}{k} \sum_{i=1}^{k} B_{i z}=\frac{1}{k} \sum_{i=1}^{k} \frac{\int_{\Omega_{i}} \rho(x, y, z) z d \Omega}{\int_{\Omega_{i}} \rho(x, y, z) d \Omega} .
$$

We know that the barycenter of the super bin using a different density distribution $\rho^{\prime}$ is higher, $S_{z}<S_{z}^{\prime}$, and want to show that the average barycenter of the $k$ bins is consequently higher too, $B_{z}<B_{z}^{\prime}$, i.e.:

$$
S_{z}=\frac{\sum_{i=1}^{k} a_{i}}{\sum_{i=1}^{k} b_{i}}<\frac{\sum_{i=1}^{k} c_{i}}{\sum_{i=1}^{k} d_{i}}=S_{z}^{\prime} \Longrightarrow B_{z}=\frac{1}{k} \sum_{i=1}^{k} \frac{a_{i}}{b_{i}}<\frac{1}{k} \sum_{i=1}^{k} \frac{c_{i}}{d_{i}}=B_{z}^{\prime}
$$

where $a_{i}=\int_{\Omega_{i}} \rho(x, y, z) z d \Omega, b_{i}=\int_{\Omega_{i}} \rho(x, y, z) d \Omega, c_{i}=\int_{\Omega_{i}} \rho^{\prime}(x, y, z) z d \Omega$ and $d_{i}=\int_{\Omega_{i}} \rho^{\prime}(x, y, z) d \Omega$. All values are strictly positive.
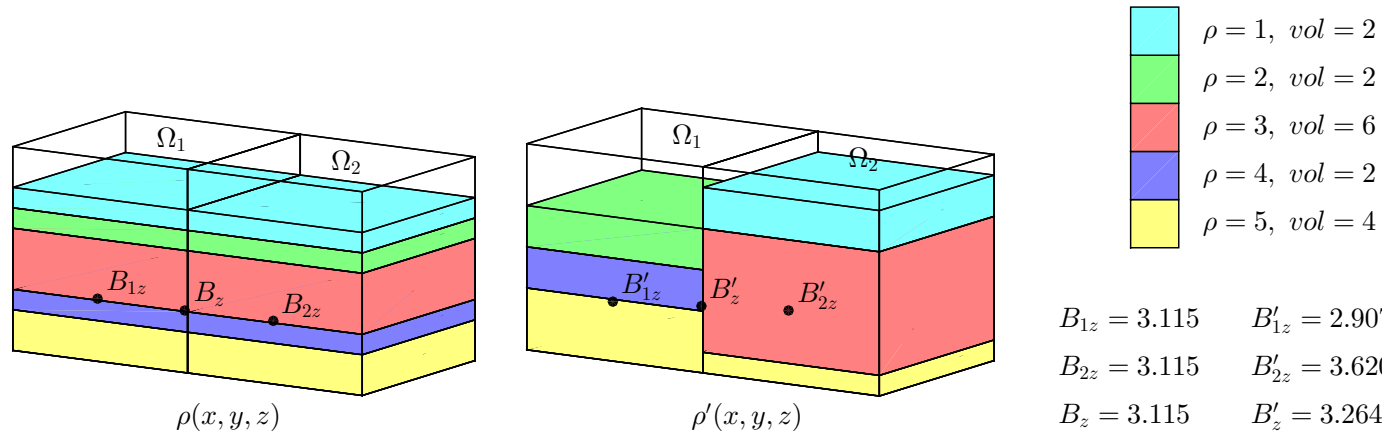

$$
\begin{array}{ll}
B_{1 z}=3.115 & B_{1 z}^{\prime}=2.907 \\
B_{2 z}=3.115 & B_{2 z}^{\prime}=3.620 \\
B_{z}=3.115 & B_{z}^{\prime}=3.264 \\
S_{z}=3.115 & S_{z}^{\prime}=3.250
\end{array}
$$

Figure 5: Five liquefied items arranged in equal layers sorted by density (left) and in a different configuration (center). The density and volume of items is indicated in the right side as well as the barycenter of individual bins $B_{1 z}, B_{2 z}$, their average $B_{z}$ and the barycenter of the super bin $S_{z}$.

Having in each bin the same configuration of layers, in the density stratified solution $a_{i}=a$ for all $i$ and $B_{z}=S_{z}$. Moreover, the total mass is preserved, i.e. $\sum_{i=1}^{k} b_{i}=\sum_{i=1}^{k} d_{i}$. The problem reduces to proving that:

$$
a<\frac{1}{k} \sum_{i=1}^{k} c_{i} \Longrightarrow \frac{k a}{\sum_{i=1}^{k} d_{i}}<\frac{1}{k} \sum_{i=1}^{k} \frac{c_{i}}{d_{i}}
$$


Set $c_{i}=a+t_{i}$, then $\sum_{i=1}^{k} t_{i}>0$ and:

$$
\frac{1}{k} \sum_{i=1}^{k} \frac{c_{i}}{d_{i}}=\frac{1}{k} \sum_{i=1}^{k} \frac{a+t_{i}}{d_{i}}=\frac{1}{k} \sum_{i=1}^{k} \frac{a}{d_{i}}+\frac{1}{k} \sum_{i=1}^{k} \frac{t_{i}}{d_{i}}>\frac{1}{k} \sum_{i=1}^{k} \frac{a}{d_{i}}=\frac{a}{k} \sum_{i=1}^{k} \frac{1}{d_{i}}
$$

Finally, the relation

$$
\frac{1}{k} \sum_{i=1}^{k} \frac{1}{d_{i}} \geq \frac{k}{\sum_{i=1}^{k} d_{i}}
$$

holds as direct consequence of the inequality of arithmetic and geometric means. This inequality states that given a list of $k$ nonnegative real numbers $r_{1}, r_{2}, \ldots, r_{k}$, the arithmetic mean of the list is always greater or equal than the geometric mean, i.e.:

$$
\frac{1}{k} \sum_{i=1}^{k} r_{i} \geq\left(\prod_{i=1}^{k} r_{i}\right)^{1 / k}
$$

Substituting $r_{i}=1 / d_{i}$ and applying the inequality of arithmetic and geometric means twice, we obtain:

$$
\frac{1}{k} \sum_{i=1}^{k} \frac{1}{d_{i}} \geq\left(\prod_{i=1}^{k} \frac{1}{d_{i}}\right)^{1 / k}=\frac{1}{\left(\prod_{i=1}^{k} d_{i}\right)^{1 / k}} \geq \frac{1}{\frac{1}{k} \sum_{i=1}^{k} d_{i}}=\frac{k}{\sum_{i=1}^{k} d_{i}}
$$

In conclusion, for any start configuration, it is possible to move to the LLB solution by decreasing the average barycenter of the $k$ bins. The LLB value is therefore the lowest possible and a valid lower bound for our optimization problem.

\section{References}

[1] M. Mongeau, C. Bes, Optimization of aircraft container loading, IEEE Transactions on Aerospace and Electronic Systems 39 (1) (2003) 140-150.

[2] S. Martello, D. Vigo, Exact solution of the two-dimensional finite bin packing problem, Management Science 44 (3) (1998) 388-399.

[3] S. Martello, D. Pisinger, D. Vigo, The three-dimensional bin packing problem, Operations Research 48 (2) (2000) 256-267. 
[11] J. Egeblad, D. Pisinger, Heuristic approaches for the two- and threedimensional knapsack packing problem, Computers \& Operations Research 36 (4) (2009) 1026-1049.

[12] A. Lodi, S. Martello, D. Vigo, Heuristic algorithms for the threesearch 141 (2) (2002) 410-420. 
[13] D. Pisinger, M. Sigurd, Using decomposition techniques and constraint programming for solving the two-dimensional bin-packing problem, INFORMS Journal on Computing 19 (1) (2007) 36-51.

[14] A. Davies, E. E. Bischoff, Weight distribution considerations in container loading, European Journal of Operational Research 114 (3) (1999) 509-527.

[15] L. Junqueira, R. Morabito, D. S. Yamashita, Three-dimensional container loading models with cargo stability and load bearing constraints, Computers \& Operations Research 39 (1) (2012) 74-85.

[16] C. Paquay, M. Schyns, S. Limbourg, A mixed integer programming formulation for the three-dimensional bin packing problem deriving from an air cargo application, International Transactions in Operational Research 23 (1-2) (2016) 187-213.

[17] B. L. Kaluzny, R. H. A. D. Shaw, Optimal aircraft load balancing, International Transactions in Operational Research 16 (6) (2009) 767-787.

[18] M. M. Baldi, G. Perboli, R. Tadei, The three-dimensional knapsack problem with balancing constraints, Applied Mathematics and Computation 218 (19) (2012) 9802-9818.

[19] T. A. de Queiroz, F. K. Miyazawa, Two-dimensional strip packing problem with load balancing, load bearing and multi-drop constraints, International Journal of Production Economics 145 (2) (2013) 511-530.

[20] A. Fernández, C. Gil, R. Baños, M. G. Montoya, A parallel multi-objective algorithm for two-dimensional bin packing with rotations and load balancing, Expert Systems with Applications 40 (13) (2013) 5169-5180.

630

[21] D. Liu, K. Tan, S. Huang, C. Goh, W. Ho, On solving multiobjective bin packing problems using evolutionary particle swarm optimization, European Journal of Operational Research 190 (2) (2008) 357-382. 
[22] A. Imai, K. Sasaki, E. Nishimura, S. Papadimitriou, Multi-objective simultaneous stowage and load planning for a container ship with container rehandle in yard stacks, European Journal of Operational Research 171 (2) (2006) 373-389.

[23] M. R. Garey, R. L. Graham, D. S. Johnson, A. C.-C. Yao, Resource constrained scheduling as generalized bin packing, Journal of Combinatorial Theory, Series A 21 (3) (1976) 257-298.

[24] C. E. Miller, A. W. Tucker, R. A. Zemlin, Integer programming formulation of traveling salesman problems, Journal of the ACM (JACM) 7 (4) (1960) 326-329.

[25] M. C. Golumbic, Algorithmic graph theory and perfect graphs, 2nd Edition, Elsevier, 2004.

${ }_{645}^{6}$ [26] R. K. Ahuja, E. Özlem, J. B. Orlin, A. P. Punnen, A survey of very large-scale neighborhood search techniques, Discrete Applied Mathematics 123 (1-3) (2002) 75-102.

[27] D. Pisinger, S. Ropke, Large neighborhood search, in: Handbook of Metaheuristics, Springer, 2010, Ch. 13, pp. 399-419.

${ }_{650}[28]$ B. Cushman-Roisin, J.-M. Beckers, Introduction to geophysical fluid dynamics: physical and numerical aspects, 2nd Edition, Elsevier, 2011. 